\title{
Jalons pour une approche socioéconomique des communs monétaires
}

Landmarks for a Socio-economic Approach to Monetary Commons

\section{Tristan Dissaux et Marie Fare}

\section{(2) OpenEdition \\ Journals}

Édition électronique

URL : http://journals.openedition.org/ei/5966

DOI : 10.4000/ei.5966

ISSN : 2553-1891

Éditeur

Association Économie et Institutions

Référence électronique

Tristan Dissaux et Marie Fare, « Jalons pour une approche socioéconomique des communs monétaires », Économie et institutions [En ligne], 26 | 2017, mis en ligne le 23 septembre 2018, consulté le 19 avril 2019. URL : http://journals.openedition.org/ei/5966 ; DOI : 10.4000/ei.5966

Ce document a été généré automatiquement le 19 avril 2019

Revue Économie et institutions 


\section{Jalons pour une approche socioéconomique des communs monétaires}

Landmarks for a Socio-economic Approach to Monetary Commons

Tristan Dissaux et Marie Fare

\section{Introduction}

1 À l'aune des crises récentes, qu'elles soient politiques ou financières, la monnaie fait aujourd'hui l'objet de nombreux débats et de multiples contestations (Ould-Ahmed et Ponsot [dir.] 2015). Une diversité de mouvements appelle d'un côté à la « libération » de la monnaie (des autorités politiques en particulier), de l'autre à sa "réappropriation » (notamment par l'État souverain). Cette opposition se cristallise clairement autour de l'euro par exemple : à l'ordolibéralisme d'une monnaie apatride et régie par des principes strictement financiers sont opposés des projets nationalistes où la monnaie servirait plus efficacement les intérêts de ses utilisateurs une fois revenue à l'intérieur des frontières des États. Cette opposition est le reflet des théories monétaires qui sous-tendent ces projets. Nous reviendrons dans une première partie sur ce corpus théorique. On y identifie assez clairement deux ensembles, en miroir de l'opposition précédente. D'une part des approches qui voient dans l'État l'initiateur et le garant de la monnaie, d'autre part des approches qui voient dans le marché l'institution la plus à même d'en garantir l'efficacité. On retrouve donc ici, en ce qui concerne la monnaie, la dichotomie marché/ État qui fonde une grande partie de l'analyse économique. Mais d'autres approches nuancent cette opposition stricte. Des travaux interdisciplinaires en sciences sociales, notamment ceux des institutionnalismes monétaires, permettent de comprendre la monnaie en tant qu'institution sociale, qui n'est alors contingente ni au marché ni à l'État.

2 Nous souhaitons ici prolonger ces approches et voir dans quelle mesure le cadre développé par Ostrom (2010) autour de ce qu'elle a défini comme des communs 
permettrait de les enrichir. Par commun, nous entendons, « un ensemble de pratiques instituantes et d'institutions constituées répondant au principe selon lequel un groupe plus ou moins étendu s'engage dans une activité collective productrice de biens tangibles ou intangibles mis à disposition des commoners ou d'une collectivité plus large, selon des règles démocratiques d'auto-organisation » (Laval 2016). De la même façon qu'on assiste dans la période récente à une "renaissance» (Bollier 2014) ou à un "retour des communs» (Coriat 2015) dans la gestion des ressources environnementales ou informationnelles, des expériences de gestion collective d'initiatives monétaires se sont développées depuis les années 1980 et connaissent une dynamique particulièrement forte depuis les années 2000. Ici, il s'agira donc d'explorer la possibilité d'instituer la monnaie en tant que tel, et donc de contribuer à "porter l'héritage de l'institutionnalisme monétaire français vers une nouvelle aventure à peine débutée » (Servet 2016).

Pour cela, nous reviendrons dans un premier temps sur les approches théoriques de la monnaie. Trois conceptions s'opposent quant aux origines et à la nature de celle-ci. La première appréhende la monnaie comme une simple marchandise, alors que la deuxième la voit comme un symbole régalien. La dernière conception, qui fera l'objet de la deuxième partie de ce texte, souligne le caractère institutionnel et fondamentalement politique de la monnaie. Cette conception nous permettra de lever le mythe d'une monnaie a-sociale et a-politique, et d'adopter une conception socioéconomique de la monnaie, ouvrant la voie à la compréhension de la pluralité monétaire. Nous proposerons enfin notre caractérisation du commun monétaire en nous appuyant notamment sur le cas des monnaies associatives. Elles constituent des exemples de projets à gouvernance participative qui mobilisent la monnaie et l'adaptent en fonction d'objectifs économiques, sociaux, et/ou environnementaux propres à leur territoire de mise en œuvre. À partir des principes qui fondent une institution commune, nous verrons de quelle façon il serait possible de conceptualiser un commun monétaire, en analysant la manière dont ces expériences de l'économie sociale et solidaire illustrent ce potentiel. Le cas des monnaies associatives permettra par ailleurs d'illustrer la possibilité de dépasser la concurrence comme seul mode d'articulation entre les monnaies, en en envisageant la complémentarité, voire la subsidiarité (Fare 2011). On verra alors que les modes de régulation des différentes monnaies ainsi imbriquées peuvent s'inscrire dans une gouvernance polycentrique au sens d'Ostrom (2011).

\section{L'appréhension du social selon les approches théoriques de la monnaie}

Différentes approches théoriques s'opposent quant aux origines et à la nature de la monnaie. Elle est le plus souvent vue soit comme une simple marchandise, soit comme un symbole régalien : la monnaie devrait par conséquent être gérée soit par le marché, soit par l'État. Outre leurs divergences quant à la nature de la monnaie, ces approches appréhendent également le corps social de manière opposée, tant quant à sa nature qu'à sa place vis-à-vis des dynamiques monétaires. Dans un cas, le social est nié car réduit à l'agrégation des préférences individuelles. Dans l'autre, il se cristallise dans une structure étatique qui ne connaît pas d'autre forme de communauté que celle de l'État-Nation. Nous revenons ici sur les grandes lignes de ces approches ainsi que sur leurs implications. 


\subsection{La monnaie au prisme du Marché : le social réduit aux individus}

5 L'approche standard de la monnaie en fait d'emblée une création du marché. Selon les origines historiques qui lui sont attribuées, ce sont les agents qui, pour dépasser les limites du troc (et notamment la contrainte de double coïncidence des besoins), inventent la monnaie dans le but de faciliter les échanges, et ainsi répondre à un besoin privé. La monnaie n'est alors qu'un bien issu de l'espace marchand, un voile posé sur les échanges. Exprimée par Say (pour qui la monnaie est un voile car les produits s'échangent contre des produits) et Jevons, cette vision pose la dichotomie entre les sphères dites réelle et monétaire. La monnaie n'est alors qu'une marchandise parmi d'autres; elle n'est désirée par les individus que dans le but de réaliser des échanges.

6 Cette origine supposée privée de la monnaie est vue comme un argument en faveur d'une gestion privée de celle-ci, car la monnaie est, dans ce cadre, le résultat du choix optimal d'agents économiques rationnels. L'accent est mis sur le caractère autorégulateur du marché, y compris celui de la monnaie, et sa gestion par ce biais est réputée être la plus efficace car exempte des interférences générées par les décisions politiques des États ou des banques centrales. Trois approches peuvent ici être distinguées.

7 La première, celle du free banking, est développée par Selgin et White (1994). Ce système de la «banque libre » repose sur une mise en concurrence des banques sans contrôle ni banque centrale et s'appuie sur une seule règle: la définition d'une unité de compte commune. Les banques sont libres d'émettre des billets et des dépôts convertibles à un taux de un pour un en une monnaie externe telle que la monnaie métallique. Cette monnaie externe est le moyen de règlement ultime et les monnaies bancaires constituent des moyens de paiement. La monnaie externe, gardienne de la régulation du système, demeure hors concurrence, alors que les monnaies internes sont en situation de concurrence. Dans cette approche, la banque centrale doit être supprimée et l'ensemble du processus d'émission monétaire laissé aux banques commerciales concurrentes. Mais cela repose tout de même sur la définition d'une norme, une unité de compte commune, qui suppose une extériorité au marché : l'État. Cette unité de compte est définie par un rapport fixe à un étalon marchandise pour rendre possible les conversions entre les monnaies internes et la monnaie externe. Le politique ainsi évincé via la suppression de l'émission de la monnaie par l'État et sa banque centrale, revient par la fenêtre lorsqu'il s'agit de définir une unité de compte et son étalonnage. Cela constitue un choix politique qui s'impose à l'ensemble des banques concurrentes d'un territoire donné et qui a pour but d'assurer la cohésion du système.

8 La seconde approche formulée par Hayek $(1976,1979)$ repose sur un principe de concurrence radicale, non plus seulement entre des monnaies internes émises par les banques, mais également sur la concurrence complète entre les systèmes monétaires afin de dénationaliser l'émission monétaire et de mettre le marché au cœur du processus. En ce sens, cette mise en concurrence repose à la fois sur les moyens de paiement mais également sur les unités de compte. Tout émetteur privé ou public peut définir sa propre unité de compte, dans laquelle est libellée sa monnaie. Des taux de change fluctuants permettent de déterminer la valeur de chaque monnaie, et chaque agent choisira la monnaie qui lui apportera la meilleure stabilité de son pouvoir d'achat. Les monnaies sont alors des marchandises soumises au mécanisme autorégulateur et spontané du marché par le biais des variations de leur prix. La régulation monétaire passe par la 
gestion de la quantité de monnaie mise en circulation par la banque émettrice, dont l'objectif est de maintenir la stabilité du pouvoir d'achat de sa monnaie. La légitimité des monnaies ainsi mises en concurrence dépend donc de cet objectif de stabilité, qui doit maintenir la confiance, attirer les dépôts et encourager les emprunts. Pour Hayek (1976), ce mode de régulation quantitatif permet de réguler l'offre de crédit (via la création ou la destruction de monnaie) et annule toute distorsion des prix relatifs provenant de la politique monétaire. Cependant, Hayek refuse de voir en la monnaie un voile neutre posé sur des échanges réels, contestant ainsi la dichotomie monétaire/réel. Mais dans sa conception, la monnaie est considérée comme un élément perturbateur par nature comme l'État - qui empêche le marché d'assurer la coordination des actes économiques. L'objectif théorique que Hayek s'assigne dès lors est de tendre vers sa neutralisation en la confiant totalement au marché.

La troisième approche, celle de la New Monetary Economics (Black 1970 ; Fama 1980 ; Hall 1981) plaide pour des systèmes monétaire, bancaire et financier totalement dérégulés et des systèmes monétaires parfaitement concurrentiels. Si l'État conserve la responsabilité de l'unité de compte, il n'existe pas de monnaie externe (contrairement au free banking). Les moyens de paiement privés seraient convertibles dans le panier de biens que l'État a choisi pour définir l'unité de compte. Pour cette école, la monnaie existe du fait de restrictions légales qui empêchent sa mise en concurrence avec d'autres actifs financiers. Par conséquent, il s'agirait d'abandonner ces restrictions légales pour favoriser une libre régulation de l'émission monétaire, et en particulier la suppression des banques centrales. L'aboutissement de cette théorie est une économie dans laquelle la monnaie telle qu'on l'entend habituellement est progressivement éliminée et remplacée par des actifs non ou peu risqués, portant un taux d'intérêt. La monnaie ne devenant alors qu'un sous-produit de la finance. Une économie sans monnaie est le point d'aboutissement de l'humanité dans cette perspective évolutionniste. Les moyens de paiement sont donc en concurrence pure et parfaite entre des banques privées émettrices exclusives de ces instruments de paiement, qui sont soumis au principe de rendement. Le paiement se réalise par le biais des banques: les agents détiennent des parts de fonds communs de placement et effectuent leurs paiements par ce moyen. Il y a compensation interbancaire des dettes et créances, jusqu'au paiement ultime des soldes.

$\mathrm{Au}$ final, ces théories n'analysent la monnaie qu'en l'assimilant à une marchandise. Celleci doit alors être placée dans un cadre concurrentiel et faire l'objet d'une régulation naturelle par le marché, à l'instar de celle qui règne sur les marchés des autres marchandises. Mais ces approches ne permettent de penser ni l'origine historique réelle de la monnaie, ni de l'appréhender au-delà de ses seules fonctions. De plus, l'ordre marchand dans lequel est supposée émerger la monnaie est fondamentalement un ordre individualiste qui n'est régie que par une logique contractuelle. Ainsi, même si l'émergence de la monnaie viendrait d'un accord unanime du corps social, ce corps n'existe pas en tant que tel au-delà de l'agrégation des décisions individuelles, ellesmêmes guidées par un principe de maximisation de l'utilité. La totalité sociale est ainsi subordonnée aux individus. Orléan (1998), analysant les théorisations de la monnaie opérées dans le cadre du marché, parle donc de «monnaie autoréférentielle », qui est pour lui « une monnaie purement instrumentale qui acquiert sa qualité de monnaie sur la base d'une représentation d'elle-même en tant que pur instrument ». Et il ajoute : « aucun point de repère extérieur à l'économie, aucun projet politique, aucune valeur sociale n'y 
sont appelés pour en légitimer l'existence. Seul l'intérêt direct des échangistes y est pris en compte».

\subsection{La monnaie au prisme de l'État : le social réduit à la Nation}

11 Si l'approche standard voit dans les forces du marché l'origine de la monnaie, des approches concurrentes justifient quant à elles du rôle de l'État dans l'organisation du système monétaire en lui attribuant une place centrale au sein de celui-ci.

12 La première approche à adopter ce point de vue est celle du chartalisme. On retient notamment le nom de son fondateur, Georg Friedrich Knapp, avec son ouvrage The State Theory of Money (1924), qui s'inscrit en rupture avec le métalisme de l'époque. Pour lui, «La monnaie est une création du droit et, en dernier ressort, peut continuer à exister même sans métaux monétaires, car l'unité de valeur est définie non techniquement mais légalement» (ibid. p. 296). Selon l'approche chartaliste, la politique fiscale de l'État est ce qui fonde en premier lieu la monnaie. Celle-ci est adoptée et utilisée en tant que telle car c'est le moyen unique permettant de régler ses dettes envers la puissance publique, le moyen permettant de s'acquitter de l'impôt. La monnaie est en outre créée par l'État lorsqu'il règle ses dépenses, et détruite par ses prélèvements. La monnaie est bien ici de «cours légal »: la monnaie n'a pas de valeur intrinsèque mais c'est l'État qui décide de l'attribution de cette valeur. Une approche qui, pour les classiques, a quelque chose de «métaphysique » (Lerner 1947) dans la mesure où la monnaie n'est «que» fiduciaire. Mais « si l'État est prêt à accepter la monnaie proposée en paiement de ses taxes et autres obligations le tour est joué» (ibid., nos italiques). Dans cette approche, seule la "State money» est reconnue comme monnaie, et le seul émetteur possible est l'autorité monétaire officielle. Tout instrument de paiement non officiel circulant sur le territoire national n'est ainsi pas reconnu comme étant de la monnaie (Bell 2001; Lerner 1947; Wray 1990). Keynes, dans le Traité sur la Monnaie (1930), s'inscrit clairement en faveur de l'approche chartaliste :

[...] l'âge du chartalisme ou de la monnaie étatique a été atteint lorsque l'État a revendiqué le droit de déclarer quelle chose devrait répondre au nom de monnaie à la monnaie de compte en vigueur - quand il a revendiqué le droit non seulement d'imposer le dictionnaire, mais aussi d'écrire le dictionnaire. Aujourd'hui toute monnaie civilisée est, au-delà de la possibilité de désaccord, chartaliste. (ibid., p. 4)

Malgré cette approbation donnée par Keynes au chartalisme, ce courant s'est quelque peu essoufflé, avant de revenir dans le débat économique à la suite de la Grande Dépression. C'est alors l'objectif du Chicago Plan que de redonner aux États le contrôle sur l'émission de monnaie: "Le Chicago Plan fournit les grandes lignes pour une transition d'un système de monnaie basée sur la dette privée à un système de monnaie émise par le gouvernement et sans dette » (Benes et Kumhof 2012).

S'il a été formulé pour la première fois par le chimiste Frederick Soddy, c'est le nom d'Irving Fisher qui reste associé à cette idée qui sera poussée auprès de l'administration Roosevelt. Elle consiste à ce que les banques de second rang soient soumises à une contrainte de « $100 \%$ réserves » (Fisher 1936), c'est-à-dire à ce que l'ensemble des crédits qu'elles octroient soit couvert par une quantité au moins égale de monnaie centrale, dont le volume est déterminé par un organe gouvernemental. L'émission monétaire serait donc centralisée au niveau de l'État. L'évolution de la quantité de monnaie centrale devrait être telle qu'elle permette la stabilisation des prix. En dépit de l'intérêt de 
l'administration Roosevelt pour ce projet, il ne fut pas adopté en tant que tel, à cause d'une forte résistance de la part de l'industrie bancaire, mais il joua un rôle majeur dans l'adoption du Banking Act en 1935 (Benes et Kumhof 2012).

Les monétaristes s'inscrivaient aussi dans l'approche de l'École de Chicago, mais avec des préoccupations différentes que celles qui avaient motivé le Chicago Plan. Pour ceux-là, la masse monétaire doit également être contrôlée par l'État, mais ce contrôle doit être séparé de toute possibilité d'action discrétionnaire. Pour Friedman notamment, et sur la base de la théorie quantitative de la monnaie, la masse monétaire devrait croître à un taux constant, indépendamment de toute politique volontariste, et notamment contracyclique (Friedman 1959). Cette règle était réputée comme devant permettre d'éviter les perturbations économiques et de garantir un niveau des prix constant sur le long terme. Elle devait aussi permettre d'éviter l'incertitude des investisseurs vis-à-vis des effets de politiques monétaires discrétionnaires, et d'échapper au comportement procyclique de la masse monétaire (Cagan 2008). On comprend ces motivations, compte tenu de la place qui est donnée aux gouvernements dans la réflexion des monétaristes. Pour eux, en matière de monnaie et de banque, « se tourner vers le gouvernement comme alternative [au strict laissez-faire] était un remède qui était pire que le mal » car «les défaillances du gouvernement pourraient être pires que les défaillances de marché " (Friedman et Schwartz 1987). Dans ce cadre, si la monnaie doit être contrôlée par l'État, elle doit dans le même temps être protégée de toute possibilité de manipulation par celuici, qui est plutôt vu comme source d'instabilité et d'inefficacité : les gouvernements produiraient d'importantes fluctuations et de l'inflation en abusant nécessairement des leviers de la politique monétaire. Ce contrôle public de la monnaie est donc, chez les monétaristes, plus technique que véritablement étatique.

Finalement, l'approche chartaliste a connu un renouveau récent avec la «Théorie moderne de la monnaie ». Plus connue sous son acronyme anglais de MMT (Modern Money Theory), cette approche néochartaliste place au centre de l'analyse le caractère souverain des systèmes monétaires :

Un système monétaire souverain est celui dans lequel le gouvernement émet sa propre monnaie libellée dans sa monnaie de compte. En règle générale, il dénomme ses taxes et autres obligations dans la même monnaie, et ses tribunaux font respecter les contrats dans la monnaie officielle de compte. (Wray 2015)

Les théoriciens de cette approche prônent donc la remobilisation par les États des possibilités de politiques monétaires autonomes, notamment pour leur financement, qui ne devrait alors plus passer par le marché. La monnaie est considérée comme endogène, ce qui signifie que "la création monétaire est liée aux opérations normales d'une économie monétaire » (Wray 1990). À ce titre, son émission n'est pas limitée par une quelconque contrainte quantitative préexistante à son émission.

Pour toutes ces théories étatiques de la monnaie, les institutions jouent un rôle actif dans l'initiation et la gestion de la monnaie. Néanmoins, ces institutions ne sont pensées qu'à l'échelle nationale, le cadre de l'État-Nation étant le seul considéré comme pertinent pour la question monétaire. La monnaie est, pour l'autorité publique, « un bien public qui confine au sacré » (Orléan 1998), de telle sorte que cette autorité exerce un contrôle strict sur la monnaie.

L'ensemble de ces approches théoriques, qu'elles fassent du marché le meilleur vecteur de l'efficacité de la monnaie, ou qu'elles considèrent l'État comme le garant de sa stabilité, ont cela en commun qu'elles voient dans les relations entre différentes 
monnaies des relations de concurrence: deux monnaies ne pourraient qu'être rivales l'une envers l'autre lorsqu'elles circulent sur un même territoire. Dans l'approche de la monnaie par l'État, et à l'intérieur des frontières nationales, toute autre monnaie que celle de cours légal, définie et imposée par l'État, n'est pas reconnue en tant que monnaie et peut être jugée comme illégale et devant disparaitre. La coercition étatique impose l'unicité monétaire. Au niveau supraétatique, chaque monnaie nationale est en concurrence pour devenir monnaie de règlement international. Dans l'approche de la monnaie par le marché, la concurrence est au cœur même du processus d'émergence de celle-ci : c'est bien la concurrence qui sélectionne la «meilleure » monnaie et qui fait que les autres, nécessairement sous-optimales comparées à celle qui a été élue par le marché, ne sont pas adoptées. À l'inverse, si une monnaie concurrente s'avère devenir plus efficiente que la monnaie en circulation, alors elle est vouée à la supplanter. En conclusion de cette revue des principales théories relatives à la monnaie, nous souhaitons donc souligner que les approches standards amènent à une appréhension restrictive du social, qui ne permet pas d'envisager des formes de gestion de la monnaie qui ne soient pas purement privées ou strictement publiques. Dépasser ces théories s'avère donc nécessaire, d'abord pour être en mesure de comprendre les dimensions plurielles de la monnaie, ensuite pour rendre compte des formes émergentes d'organisation collective autour de la monnaie.

\section{Dépasser la dualité État/Marché : la monnaie comme institution sociale}

21 Si selon les approches précédentes, toute forme de politique est effacée, ou au contraire placée en position d'hégémon, la monnaie n'est pour autant pas contingente aux sociétés étatiques, pas plus qu'elle ne relève de pures dynamiques de marché. La monnaie n'est pas qu'un instrument économique, mais elle est aussi une institution dont les dimensions politiques et symboliques sont cruciales pour qu'elle soit acceptée en tant qu'instrument économique. Dans le même temps, elle intervient comme moyen de médiation dans des activités multiples, y compris au sein de sphères non marchandes, et ne saurait être réduite à un actif financier ou à une marchandise. C'est ce que soulignent de nombreux travaux interdisciplinaires sur la monnaie incluant notamment les apports de l'histoire ou de l'anthropologie. La monnaie apparaît alors comme antérieure au marché tout comme à l'État. En effet, la monnaie est d'abord une institution sociale au fondement de toute société. Polanyi (2008b) souligne la présence de pratiques monétaires bien avant l'utilisation de la monnaie en tant que moyen d'échange. Il n'existerait donc pas de société sans monnaie dans la mesure où la monnaie est constitutive de la dette et que les liens de dette - entre les individus eux-mêmes et entre les individus et la totalité sociale sont les fondations de toute société. Étudier la monnaie nécessite donc de dépasser un cadre réducteur qui en ferait un simple instrument économique des échanges marchands.

\subsection{La conception polanyienne de la monnaie}

Pour déconstruire la conception dominante de la monnaie, un détour par Polanyi semble salutaire. Polanyi (2008a [1957], 2008b [1957]), dans des textes majeurs, a probablement contribué de manière fondamentale à remettre en cause les conceptions traditionnelles de la monnaie et de son origine en soulignant notamment la présence de la monnaie dans 
les sociétés anciennes : « certains usages de la monnaie sont aussi vieux que l'humanité » (Polanyi 2008a [1957], p. 65). De fait, au sein des sociétés anciennes et bien qu'il ait pu y être présent, le troc n'a jamais constitué un principe d'organisation économique, comme l'affirment les approches adoptant une conception instrumentale de la monnaie. Polanyi (2008a [1957]) fonde son approche en termes d'usages monétaires (money uses) qui peuvent prendre trois dimensions: le paiement (extinction d'une obligation), le rôle d'étalon (unité de compte) et l'échange. "La monnaie est donc définie ici en termes d'objets quantifiables qui sont employés pour un ou plusieurs de ces usages » (Polanyi 2008a [1957], p. 72). Il établit ensuite une distinction entre «monnaie à tous usages » (all purpose money) et "monnaie à usages spécifiques " (special purpose money). Les premières caractérisent les sociétés modernes dans lesquelles la monnaie est utilisée de façon concomitante pour ses trois usages et les secondes seraient l'apanage des sociétés anciennes dans lesquelles différents objets sont utilisés pour différents usages de la monnaie. Dans ce cas, « les différents usages de la monnaie sont donc institutionnalisés de façon séparée » (Polanyi 2008b [1957], p. 234).

Polanyi affirme avec vigueur ce que Servet (2001) appellera la «fable du troc»: «le commerce et la monnaie ont des origines séparées et indépendantes des marchés. Ils ne naissent pas, comme on l'a longtemps pensé, du troc individuel et de l'échange » (Polanyi 2008b [1957], p. 233). La monnaie est logiquement antérieure au marché : elle « remonte à fort loin dans l'histoire de l'humanité et on en retrouve la trace dans la plupart des sociétés » (Théret 2008, p. 814). Elle ne constitue donc pas une caractéristique des sociétés modernes. Au contraire, Polanyi met en exergue le fait que l'endettement puisse avoir une nature non économique liée au statut, à la filiation, au prestige, aux alliances, au sacré, ou aux rites. En ce sens la monnaie est avant tout un moyen de paiement. La monnaie comme moyen d'échange aurait donc une origine plus tardive que la monnaie comme moyen de paiement et résulterait du commerce extérieur. Il en conclut qu'une définition substantive de la monnaie doit être indépendante du marché. Cette définition découle des usages déterminés que l'on attribue à des objets quantifiables.

Ainsi, l'analyse de Polanyi souligne d'une part qu'il est nécessaire de distinguer monnaie et marché, et donc de ne pas la penser exclusivement dans ses liens à celui-ci et, d'autre part, l'universalité du fait monétaire. En revanche, la conception polanyienne ne permet pas de rompre véritablement avec la conception standard de la monnaie selon laquelle la monnaie assume ensemble les fonctions de compte, de paiement et de réserve (du moins pour les sociétés dites modernes qui connaissent des monnaies à tous usages), et l'idée selon laquelle la monnaie permet d'accéder à l'ensemble des biens et services disponibles, avec pour corollaire le principe de fongibilité de la monnaie (Blanc 2009). Qui plus est, elle conduit à considérer que les sociétés modernes ne connaissent pas de special purpose money. C'est en ce sens que des travaux socioéconomiques visent à dépasser l'approche polanyienne pour rendre visible la pluralité tant dans les usages monétaires que dans le type de monnaies en adoptant une approche par les pratiques ou usages monétaires.

\subsection{Penser la pluralité monétaire}

25 À l'encontre de l'homogénéité que les théories standards confèrent à la monnaie, son étude par la socioéconomie révèle qu'elle peut être différenciée de plusieurs façons, et notamment revêtir différentes qualités. En premier lieu, «l'argent prend la couleur des personnes, organisations ou institutions dont il émane » (Blanc 2009). En fonction de 
l'autorité émettrice, ou bien de l'organisation ou de la personne desquelles la monnaie est reçue, elle sera chargée d'un sens différent. Mais il peut aussi lui être associé des valeurs morales particulières, et elle peut être confinée à une sphère sociale spécifique. L'étude des pratiques monétaires permet donc de voir que la monnaie circule au sein d'univers symboliques multiples, chacun ayant son propre système de normes.

Ce qui est valable pour une même monnaie l'est évidemment d'autant plus lorsqu'on considère la pluralité des moyens d'échange générée en particulier par la mise en place de monnaies associatives (deux outils monétaires libellés en la même unité de compte pouvant alors circuler dans deux sphères d'échange distinctes en même temps qu'ils circulent sur un même territoire). Cette pluralité, conjuguée avec cette différenciation, remettent alors en cause le postulat économiciste de la fongibilité généralisée des avoirs monétaires : « les qualités des avoirs rendent la fongibilité improbable, voire impossible ; ceci a pour conséquence de réduire leur substituabilité, et donc la capacité de concurrence qui est au cœur des raisonnements économistes » (ibid.). Le mode de relation des monnaies entre elles mis en avant par les approches standards, à savoir la concurrence, n'est donc pas le seul principe possible. Deux monnaies peuvent en effet être complémentaires si elles servent des échanges de types différents à l'intérieur de sphères d'échange distinctes. Elles peuvent de plus être subsidiaires si ces sphères d'usages diffèrent non seulement du point de vue de leurs usages mais également d'un point de vue territorial (Fare 2011, 2018).

L'observation des pratiques monétaires fait finalement apparaître le caractère fragmentaire de la monnaie, et non plus son hypothétique caractère plein ou unifié : la monnaie s'incarne en une série d'instruments divers et hétérogènes. Repenser la diversité de la monnaie suppose ainsi de sortir d'une perspective d'unicité monétaire pour appréhender la pluralité historique des pratiques et des formes monétaires. À l'époque des monnaies métalliques par exemple, on observait clairement la différenciation des espaces de circulation d'espèces monétaires diversifiées : monnaies de cuivre ou de billon circulant à l'échelle des communautés villageoises, monnaies d'argent pour les échanges nationaux et la fiscalité, et monnaies d'or pour les échanges internationaux (Courbis, Froment, Servet 1990 ; Blanc 1994). Les crises expriment ce fait récurrent qu'une seule et même monnaie ne peut que très mal remplir simultanément des usages diversifiés, voire ambivalents (Théret [dir.] 2007), dans la mesure où seule la pluralité monétaire peut y répondre.

Cette pluralité, loin d'être l'apanage des sociétés anciennes est également un fait constitutif des sociétés contemporaines. En effet l'étude des usages monétaires souligne, d'une part, l'existence d'usages monétaires différenciés (Zelizer 2005 [1994]) ${ }^{1}$ et d'autre part, l'existence de formes et de monnaies diverses (comme les monnaies associatives), qui font apparaître les diverses qualités des monnaies: la forme prise par les avoirs monétaires, l'univers symbolique dans lequel ils s'insèrent et les marqueurs socioéconomiques qui orientent les usages sociaux de la monnaie (Blanc 2009). La forme monétaire prise par les avoirs constitue la première forme de différenciation : il s'agit des pièces, billets et monnaies bancaires, porte-monnaie électronique, bons d'achats, chèques cadeaux, monnaies affectées telles que les chèques déjeuner ou chèques livre, les monnaies locales et les systèmes de fidélisation. Il existe donc une diversité de formes monétaires permettant de différencier les avoirs monétaires. Ensuite, chacun d'eux circule dans un univers symbolique spécifique, c'est-à-dire un «espace homogène de représentations caractérisé par une hiérarchie en valeurs et en normes morales [...] qui 
dispose de sa propre légitimité et de ses propres modes de légitimation » (Blanc 2008a, p. 33). L'univers symbolique se réfère aux représentations sociales, aux valeurs et aux normes qui fondent l'adhésion collective à la monnaie. Plusieurs univers symboliques peuvent exister au sein d'une même société, et ainsi ouvrir des espaces de circulation monétaire distincts. Enfin, les marqueurs socio-économiques relèvent de « procédures de différenciation des avoirs dans leurs usages, indépendamment de la forme monétaire que ces avoirs prennent » (Blanc 2008a, p. 34). Ces marqueurs orientent les usages sociaux de la monnaie, ce sont des moyens de différenciation subjective s'ancrant dans des facteurs d'ordre cognitif et de normes morales. La mise en exergue de ces critères qualitatifs de différenciation, dont disposent les avoirs monétaires, corrobore donc la thèse de la pluralité monétaire et infirme celle de la fongibilité pure. Ainsi «l'examen des conditions de différenciation et de conversion de la monnaie permet une déconstruction du postulat économiste de la fongibilité. La fongibilité apparaît ainsi plurielle » (Blanc 2009a, p. 677).

\subsection{La monnaie, un fait social total}

La monnaie peut être appréhendée en tant que « fait social total » au sens de Mauss (1995 [1923-1924]) car elle touche à l'ensemble des sphères sociale, politique, culturelle, religieuse, symbolique et économique. L'approche la plus aboutie, à notre sens, de la démonstration de la nature de la monnaie en tant que fait social total est développée par Théret (dir., 2007 et 2008), à la suite des travaux d'Aglietta et Orléan (dir., 1998). L'intérêt de l'approche de Théret (2007) est de compléter la «déconstruction» de la vision instrumentale de la monnaie en rompant fondamentalement avec l'approche en termes de fonctions monétaires par la combinaison de l'appréhension de la monnaie comme rapport social et comme fait social total. Cela tend à renforcer les approches institutionnalistes en leur apportant une véritable cohérence.

En effet, Théret (2007) distingue "les trois états de la monnaie » à partir non de ses fonctions mais de l'appréhension de la monnaie comme un «lien social universel de nature économique, politique et symbolique » (ibid. p. 38) ce qui permet de faire émerger la nature de la monnaie et son fonctionnement spécifique en tant que fait social total. Le premier état, la «monnaie incorporée » souligne qu'elle « est présente dans la personne même de ses utilisateurs, qu'elle fait partie de leurs habitus, qu'elle est inscrite dans leur système de dispositions incorporées, la confiance se jouant en chaque individu » (ibid. p. 43). Dans cet état, la monnaie apparaît comme étalon de valeur et confiance. En ce sens la monnaie représente un langage, un «système symbolique " permettant aux individus d'une même société de communiquer et d'échanger dans une relation de confiance. La monnaie représente un signe par lequel «des symboles et des significations partagées sont échangés » et apparaît ainsi comme un « opérateur de l'appartenance sociale » (ibid. p. 43). Confiance éthique et confiance méthodique sont au cœur de la monnaie incorporée et se renforcent mutuellement. La première renvoie au système de compte, elle est le signe de l'appartenance à une communauté et à un ensemble de valeurs et de représentations au cœur de l'appartenance sociale. La deuxième renvoie aux moyens de paiement, et est le signe de l'usage routinier de la monnaie dans les échanges. Partant de ces deux formes de confiance, l'appréhension de la monnaie comme système symbolique conduit à ne la percevoir que comme une relation horizontale. Or, comme l'a souligné précédemment l'ouvrage d'Aglietta et Orléan (dir., 1998), la monnaie en tant qu'objet d'appropriation, de contrôle et donc de pouvoir, doit être considérée comme légitime. 
Intervient ici la confiance hiérarchique relevant d'un tiers et qui établit les règles monétaires et ainsi régule et protège afin de garantir la cohésion sociale. La monnaie met simultanément en relation des «individus entre eux et avec des entités collectives représentant la souveraineté du groupement d'appartenance » (ibid. p. 38). La monnaie ne peut donc être découplée de ses dimensions horizontale et verticale: confiance et monnaie sont intimement liées, l'acceptation généralisée de la monnaie repose en premier lieu sur la confiance.

31 Le deuxième état, la « monnaie objectivée », repose sur les instruments monétaires qui servent de moyen de paiement. En effet, si la confiance constitue pour Théret (ibid.) une " précondition » à l'usage de la monnaie, elle nécessite aussi des moyens de paiement qui se caractérisent par trois dimensions : deux faces, dont une représente sa valeur et l'autre le symbole de l'autorité émettrice, et une tranche qui représente le monnayage.

Le troisième état, la "monnaie institutionnalisée ", capte les règles unifiant un espace monétaire et dans lequel la monnaie représente « la forme politique d'une communauté de paiement qui n'est autre que le tout social représenté sous forme monétaire " (ibid. p. 48). Pour former une communauté de paiement, les membres doivent: 1) reconnaître un système de compte permettant de quantifier dettes et créances et qui symbolise l'expression de la totalité ; 2) où circule un ensemble de moyens de paiement liant les membres de la communauté et confortant l'appartenance sociale; et 3) instituer des règles de compte et de paiement qui permettent la constitution de la communauté, sa pérennité et sa reproduction.

Par conséquent, ces approches institutionnalistes soulignent la nécessité de rompre avec une approche de la monnaie qui ne serait qu'économiciste. En termes plus politiques, cela implique d'abandonner une approche technocratique de la monnaie, pour penser non seulement les possibilités de décentralisation du pouvoir monétaire, mais également de réintroduire de la démocratie dans les choix monétaires. Cela permettrait dès lors d'envisager le rôle de la société civile. Ces approches ouvrent des perspectives pour penser la monnaie comme commun, en partant de la double dimension verticale et horizontale de la monnaie tout en rejetant une vision exclusive de la souveraineté monétaire.

Cette question de la souveraineté est cardinale et demande ici à être précisée. La souveraineté monétaire peut être définie comme l'exercice d'un pouvoir dans le domaine monétaire, qui le plus souvent est entendu comme un pouvoir exclusif. Donc comme le "résultat de la déclaration de souveraineté d'une autorité se donnant la prérogative exclusive de définir l'unité de compte, de définir le revenu de souveraineté qu'elle souhaite prélever et de définir les marques de la souveraineté »(Blanc 2002). Les approches théoriques standards de la monnaie sur lesquelles nous sommes revenus dans la première partie de ce texte emportent avec elles une conception de la souveraineté monétaire qui est exclusive: que l'État joue un rôle actif ou non au sein du système monétaire, la monnaie doit relever d'un pouvoir unique et plein.

Mais en abandonnant un point de vue strictement économiciste sur la monnaie, en prenant au sérieux son caractère d'institution sociale, et en en comprenant sa pluralité, cette conception de la souveraineté peut être remise en cause. L'étude des pratiques monétaires invite en effet à « opérer une dissociation épistémologique, sinon ontologique entre souveraineté politique et souveraineté monétaire" (Blanc 2013). La monnaie circulant au sein d'univers symboliques multiples pouvant être distincts - chacun d'eux ayant son propre système de normes - s'y exerce alors au sein de chacun une forme 
propre de souveraineté. La souveraineté ne doit ici plus être entendue comme émanant exclusivement du souverain en tant que prince, autorité suprême, ou gouvernement central. Mais il y a expression d'une forme de souveraineté monétaire dès lors que des individus sont mis en lien par l'usage d'une monnaie, qu'ils constituent une communauté monétaire. Théret et Zanabria (2007) montrent bien, à travers l'étude du cas argentin et du fédéralisme monétaire qui y a prévalu de façon récurrente, que «la souveraineté monétaire et la souveraineté politique ne doivent pas être confondues ». Des formes de souveraineté monétaire d'ordre ou d'échelle inférieure à celle de la monnaie nationale peuvent donc s'exercer, par exemple à travers l'établissement de monnaies associatives. Des groupes institués de la société civile génèrent alors des formes de souveraineté monétaire subsidiaire à celle de la monnaie nationale. On peut alors parler de souverainetés imbriquées.

Reconnaître cette capacité à d'autres formes d'organisation que les seules formes gouvernementales ou administratives amène donc à envisager la place de collectifs de la société civile, dont les initiatives ne sont pas purement privées pour autant. Elles peuvent en effet prendre la forme d'organisations communes. C'est cette possibilité que nous souhaitons explorer dans la section suivante.

\section{Socioéconomie du commun monétaire}

Pour envisager le caractère commun de la monnaie, il nous faut d'abord préciser la nature de cet objet. La théorie économique, à la suite de Samuelson (1954), retient les critères de rivalité et d'exclusion pour établir une typologie des biens, chacun des quatre types définis par le croisement de ces deux critères étant associé à des modes de provision et de gestion spécifiques. Mais appliquer cette grille à la monnaie fait d'emblée apparaitre son caractère singulier et fait se manifester son ambivalence. Une fois soulignée la nature politique, symbolique et éthique de la monnaie, il est aisé d'en comprendre également l'ambivalence: à la fois système de règles pour assurer la coordination au sein de la société, et objet susceptible d'être approprié privativement par chacun des individus la composant (Aglietta et al. 2016). En effet, les agents recherchent la liquidité et la monnaie fait alors l'objet du désir illimité de tous dans la mesure où elle permet d'accéder à l'ensemble des biens et services. Il y a donc ici un paradoxe qualifié d'ambivalence de la monnaie (Aglietta et Orléan 1982) lié à l'incompatibilité entre son appropriation individuelle et l'entretien d'une liquidité collective: son appropriation limitant les liquidités disponibles pour le bon fonctionnement de l'économie et des sociétés. Le compromis social et politique sur lequel repose la monnaie et qui est fondé sur la confiance sous ses différentes formes est donc essentiel à la régulation de cette ambivalence. Avoir confiance dans la monnaie, c'est reconnaitre un collectif, les règles mises en œuvre en son sein, ses valeurs, et sa légitimité à réguler la monnaie : «La confiance dans la monnaie est une attitude collective, donc une institution implicite, qui conjure les forces corrosives de l'ambivalence » (Aglietta et al. 2016).

Par conséquent, il y a une opposition forte entre la finalité première de la monnaie, qui est de permettre de régler et/ou d'honorer les dettes/créances créées par les échanges sociaux (dont la création est elle-même stimulée par l'existence d'instruments monétaires), et l'usage capitaliste qu'il est possible d'en faire en la soustrayant des circuits de ces échanges pour soit la conserver par devers soi en tant que valeur inaltérable, pérenne et liquide, soit la faire circuler dans des circuits financiers où son 
accumulation est considérée comme une fin en elle-même. Les politiques monétaires menées à la suite de la Grande Récession par toutes les principales banques centrales du monde et en particulier par la Banque Centrale Européenne sont représentatives de cette ambivalence, puisqu'avec les mesures non conventionnelles de Quantitative Easing, on a une " politique publique de création monétaire par la banque centrale, visant à faciliter la circulation privée de la monnaie dans les canaux du crédit» (Amato 2016). Le taux d'intérêt est la variable centrale de la régulation d'une telle antinomie, puisque de la fixation de son niveau dépend l'arbitrage entre deux «fonctions » contradictoires de la monnaie - la mise en circulation des droits de propriété sur les biens et services, ou la mise en réserve et l'accumulation de richesse.

En résumé, si la monnaie est appréhendée en tant que forme de richesse unanimement désirée, alors elle est une ressource rivale et exclusive. Elle tient alors du bien privé. Mais si la monnaie est comprise comme une convention ou une institution, alors elle est nonexclusive et s'apparente alors à un bien commun. Cela dit, distinction doit être faite entre les biens communs, qui de par leur nature sont sujets à la «tragédie des communs » telle que théorisée par Hardin (1968), et les communs, qui sont collectivement institués en tant que tels indépendamment de leur nature ou de leurs caractéristiques propres. C'est donc aux modalités d'institution du commun monétaire que nous nous intéresserons par la suite. Nous souhaitons avant cela le définir plus précisément.

\subsection{Définition du commun monétaire}

On retient, à la suite d'Ostrom (2010, p. 90), huit principes de conception des communs qui sont partagés par la plupart d'entre eux : 1) des limites clairement définies pour la ressource ; 2) la concordance entre les règles d'appropriation et de fourniture et les conditions locales - règles qui restreignent l'accès à et l'usage de la ressource, en termes de temps, d'espace, de technologie; 3) des dispositifs de choix collectif; 4) la surveillance ; 5) des sanctions graduelles; 6) des mécanismes de résolution des conflits ; 7) une reconnaissance minimale des droits d'organisation ; 8) des entreprises imbriquées (dans le cas où le commun s'inscrit dans le cadre d'institutions de niveau supérieur). Cependant, ces principes étant premièrement associés aux ressources naturelles, le commun monétaire présenterait a priori des spécificités, de par la nature particulière de cette ressource. Pour ce qui est des ressources naturelles, il s'agit dans le cas d'une ressource non renouvelable de maximiser l'usage au cours du temps du stock disponible, ou dans le cas d'une ressource renouvelable de s'assurer que le prélèvement ne dépasse pas la capacité de renouvellement de la ressource. La problématique posée est donc celle de la gestion d'une quantité. Dans le cas de la monnaie, il n'existe pas de telle problématique quantitative. La monnaie est abstraite et immatérielle : c'est d'abord et avant tout de la gestion de l'institution dont il s'agit, bien plus que de celle de sa manifestation objectivée dans les moyens de paiement.

S'il n'existe pas de commun par nature, ce sont bien les pratiques sociales qui se nouent autour d'une ressource qui permettent de l'instituer en tant que tel. Toujours suivant Ostrom, ces pratiques passent par l'auto-organisation des parties prenantes, la transparence des règles, la régulation collective, la participation des acteurs, la nonappropriation individuelle du surplus, la coopération des membres, des processus décisionnels participatifs et collectifs, etc. L'analyse doit donc porter sur ces pratiques : ce sont in fine les qualités octroyées à une ressource par la mobilisation d'un «agir 
politique " qui font émerger des communs (Dardot et Laval 2015). C'est donc par sa construction sociale en tant que commun que la monnaie le devient : la monnaie est un enjeu politique (Harribey et al. 2018), et « la monnaie ne peut devenir un commun qu'au terme d'une décision politique » (Giraud 2013). Cette décision politique peut procéder de l'agir citoyen, comme nous le montrerons par la suite.

Giraud (ibid.) met premièrement l'accent sur le caractère de liquidité de la monnaie et avance que « compte tenu du fait que toute transaction marchande s'effectue aujourd'hui par la médiation monétaire, aucun citoyen ne devrait être exclu de l'accès à la monnaie ». Cette question de l'accès est également au cœur de la réflexion de Baronian et Vercellone (2015), pour qui « le caractère spécifiquement monétaire du rapport capital/travail forme le seul point de départ adéquat pour une réflexion sur la monnaie du commun». Adoptant une démarche circuitiste, ces auteurs notent qu'une classe sociale "gagne ce qu'elle dépense » pendant qu'une autre "ne dépense que ce qu'elle gagne» du fait de l'accès au pouvoir de création monétaire des premiers et de la soumission au rapport salarial des seconds. Ainsi, et pour avancer vers une définition, un commun monétaire serait un mode de gestion de la monnaie qui garantisse à chacun un accès équitable à celle-ci, compte tenu de ses obligations en termes d'échanges et de satisfaction de ses besoins. On rejoint ici des questions pouvant être liées au financement d'un revenu de base, questions qui dépassent le cadre de cette contribution (voire par exemple Arnsperger 2015).

Outre cette première dimension de la liquidité, par la monnaie et à travers ses modalités d'émission, se concrétise également une fonction de financement de l'économie. Entreprendre un investissement nécessite d'avoir accès à un supplément de monnaie, qui a pour origine principale le crédit bancaire. De la monnaie nouvellement créée est rendue disponible à l'occasion de chaque opération de crédit consentie par une banque. La question qui se pose est alors celle de la capacité d'accès des individus à ce mode de financement, et de la bonne volonté du système bancaire à assurer ce financement. Cette dernière apparaît aujourd'hui insuffisante, le système bancaire étant perçu comme ne participant pas assez au financement de l'économie réelle, ce que les chiffres tendent à confirmer: selon la Banque des Règlements Internationaux, seuls $15 \%$ des crédits bancaires servent à l'investissement. Alors que le système bancaire privé peut être vu comme ayant réalisé un processus d'enclosure de la monnaie, il n'assume plus aujourd'hui le rôle qui lui incombe, notamment dans un contexte de nécessaire transition écologique et sociale de nos sociétés. Pourtant, c'est bien le système bancaire qui autorise et oriente la création de richesse au sein de l'économie. En fonction de ce qui est effectivement financé ou de ce qui ne l'est pas, c'est la structure productive de nos économies qui est ainsi façonnée. Un crédit est émis, et donc de la monnaie est créée, en anticipation d'une création future de richesse: le point crucial est donc ici que cette émission monétaire autorise - en ce qu'elle lui est préalable - cette création de richesse. Un commun monétaire serait donc également un mode de gestion de la monnaie qui reconnaît pour toute personne ou pour toute entreprise la capacité de création future de richesse et en autorise la réalisation, et qui garantit le financement des activités jugées souhaitables du point de vue collectif et dans une perspective de long terme.

Une fois posés ces premiers éléments de définition, nous souhaitons maintenant avancer dans la conceptualisation du commun monétaire, notamment en croisant les apports d'Ostrom et de Polanyi. Nous explorerons ensuite comment l'institutionnalisation de ce 
commun peut se réaliser, en mobilisant les quelques exemples qui ont déjà fait l'objet d'une telle réflexion et en s'appuyant sur le cas des monnaies associatives.

\subsection{Conceptualisation du commun monétaire}

La mise en avant par Karl Polanyi (2008a) de plusieurs " principes d'intégration » permet de reconnaître la pluralité des logiques d'action qui peuvent coexister au sein de nos économies. En effet, les acteurs ne sont pas mus uniquement par des considérations qui feraient d'eux des êtres rationnels et maximisateurs, mais ils peuvent aussi fonder leurs décisions sur la base de critères plus larges, qui dépendent eux-mêmes de l'action considérée et du contexte dans lequel elle prend place. On retient donc qu'à côté du marché existent également des logiques de réciprocité, de redistribution, ou de partage domestique (householding).

Sans s'inscrire dans le débat sur les communs, Blanc (2013) a établi une typologie des monnaies modernes en croisant pluralité des émetteurs de monnaie et des formes d'intégration de Polanyi. Trois idéaux-types sont alors identifiés: monnaie publique, monnaie lucrative et monnaie citoyenne. Chacun de ces idéaux-types est ensuite affiné en sous-types. Pour ce qui est des monnaies citoyennes, il distingue des monnaies citoyennes communautaires (renvoyant à des logiques de reproduction de la communauté par la réciprocité et qui correspondent à des dispositifs de banques de temps ou de systèmes d'échange local) et des monnaies citoyennes commerciales (renvoyant à la distribution horizontale des biens et services par l'échange via l'usage de monnaies associatives). Si les monnaies citoyennes mobilisent potentiellement les quatre principes d'intégration polanyiens, elles s'inscrivent premièrement dans des logiques de réciprocité et de redistribution et portent « un projet solidaire qui est aussi une lutte contre des formes de domination étatique et marchande » (ibid.). Cela devra nous amener à nous interroger sur les relations complexes que ces monnaies nouent avec le marché ainsi qu'avec l'État (et par conséquent à l'articulation des différentes formes d'intégration). Cependant, comme le souligne Blanc, mais sans en préciser les caractéristiques, certaines monnaies commerciales « combine[nt] l'échange et le partage domestique » (ibid.). La mise en avant de ce quatrième principe d'intégration de Polanyi constitue une piste prometteuse pour l'analyse des communs monétaires qui doit par contre être précisée.

Servet et Swaton (2017) ont entamé cette réflexion sur le caractère de commun des monnaies locales complémentaires. Les auteurs souhaitent prolonger les travaux de $L a$ monnaie souveraine (Aglietta et Orléan [dir.] 1998), qui occulteraient selon eux le partage au profit de l'échange, du fait d'une approche principalement catallactique ou circulatoire. Servet et Swaton commencent donc par souligner (poursuivant Servet 2013) que «la réciprocité ne se confond pas avec le don»: une approche en termes de don contre-don serait restrictive alors que la réciprocité doit être "entendu[e] comme une institutionnalisation de la solidarité ». En cela, la dette, surplombant le triptyque dettesouveraineté-confiance, et à laquelle est associée la subordination, ne doit pas occulter le partage, qui a pour élément constitutif la mise en commun. Sur ces bases, il apparaît donc que « les monnaies ne sont pas nécessairement des contreparties de bien ou de service, mais, au sein de communautés, elles s'inscrivent comme institution de l'interdépendance. Chaque acte auquel la monnaie participe ne fait sens que par rapport à l'ensemble des liens qu'elle tisse, retisse et dénoue" (Servet et Swaton 2017, p.6). Les monnaies complémentaires apparaissent alors comme des vecteurs de réciprocité volontairement 
recherchée par les membres de ces dispositifs. Dans cette perspective, les pratiques de partage sont insérées dans le principe d'intégration polanyien de la réciprocité (élargi audelà du don contre-don), ce qui nous semble discutable dans la mesure où il est possible d'envisager le partage comme caractérisant le quatrième principe d'intégration de Polanyi.

En effet, le householding développé par Polanyi (1983 [1944]) ${ }^{2}$ et remis à jour par Hillenkamp (2013) à travers le concept de " partage domestique ", également qualifié de principe "d'autosuffisance» ou "d'autosatisfaction» par Servet (2015c) pourrait informer les pratiques monétaires citoyennes ${ }^{3}$. Ce principe renvoie, en suivant la piste tracée par Hillenkamp, à des interdépendances à l'intérieur d'un groupe domestique non nécessairement autarcique mais réuni par une identité commune. Cette approche permettrait de conserver la vision de Polanyi d'un principe d'intégration fondé sur la symétrie et l'interdépendance volontaire (la réciprocité) tout en élargissant le partage domestique à des formes démocratiques au-delà du seul cadre domestique. Ce principe d'intégration apparaît dans Aristote découvre l'économie (2008 [1957]) mais Polanyi n'y précise pas les caractéristiques institutionnelles à l'exception de la notion de partage : «si la logique de partage caractérise donc le principe polanyien de householding, l'autarcie ne semble pas en revanche indispensable à l'institution du groupe dans lequel il opère. Un groupe peut reposer sur des règles de production et de partage des richesses pour l'usage de ses membres, tout en participant par ailleurs à des échanges, y compris marchands : la logique de partage peut dominer tant que les ressources issues des échanges avec l'extérieur sont soumises au principe de satisfaction des besoins des membres du groupe » (Hillenkamp 2013, p. 223). Ainsi, le partage domestique "définit un principe d'intégration spécifique, qui n'est pas réductible à la réciprocité, qui se fonde sur diverses logiques d'action entre pairs, pas plus qu'à la redistribution, qui relève de l'obligation dans un système centralisé, ou au marché.» (ibid. p. 224). Comme le souligne Hillenkamp, la mise en œuvre du principe de partage domestique n'implique pas que les interdépendances entre les membres du groupe relèvent de solidarités démocratiques : le partage peut par exemple être hiérarchisé ou genré. Pour autant, le principe de partage domestique peut mener, sous certaines conditions qu'il nous faudra donc expliciter, à des formes démocratiques communautaires telles que celles mises en œuvre dans le cadre des communs. Une fois restitué dans son contexte historique et dans la tradition aristotélicienne (opposant économie et chrématistique), il semble possible d'élargir ce principe d'intégration, comme Polanyi le proposait, à un groupe partageant une identité commune et à partir duquel un partage des ressources est opéré. L'étude des pratiques pourrait alors faire évoluer les principes d'intégration polanyiens pour prendre en compte les transformations contemporaines et faire du concept de partage domestique « un concept plus général que celui introduit par Polanyi à partir du cas des sociétés agraires autarciques» (ibid. p. 236). C'est pourquoi, lorsque ce principe d'intégration mène à des formes démocratiques d'interdépendance, nous proposons de le requalifier en "partage communautaire ».

49 En ce sens, il s'agit d'analyser et de caractériser les institutions sur lesquelles repose et se réalise le principe de partage communautaire, institutions qui orientent les comportements individuels et confèrent unité et stabilité au procès économique. Ainsi, l'approche à partir des règles d'usage développées pour gérer une ressource, et proposée par Ostrom, vient compléter et s'articuler à l'analyse des principes d'intégration de 
Polanyi. Appliquée au commun monétaire, nous analyserons les règles du groupe de la communauté monétaire, permettant la mise en commune de la ressource (ici la monnaie).

Suivant l'idée polanyienne de householding ici renouvelée en partage communautaire, une des premières caractéristiques d'un commun monétaire serait donc une identité commune. Cela est fortement pointé par les monnaies associatives dans la mesure où elles activent, à travers la confiance éthique et l'univers symbolique qu'elles créent, un ensemble de valeurs propres à la communauté monétaire ${ }^{4}$ : elles prennent corps notamment dans le nom choisi pour la monnaie et dans l'iconographie des billets. La communauté ne préexiste pas à la monnaie, au contraire elle est construite par et autour de l'usage de la monnaie dans la mesure où la monnaie agit comme opérateur de lien et comme vecteur de valeurs. Elle permet donc l'institutionnalisation de cette communauté. Par conséquent, les valeurs et les règles d'usage adoptées par le groupe représentent in fine ce qui fait commun dans la monnaie. Par exemple, dans le cas des monnaies associatives, une des étapes clés dans la construction de l'identité commune d'un groupe monétaire local/citoyen repose sur le processus d'élaboration de la charte de l'association gestionnaire de la monnaie, charte qui institue le cadre des valeurs partagées par les fondateurs de celle-ci. Elle est en quelque sorte la loi fondamentale du collectif qui l'adopte, et qui préside à la mise en place des modalités de fonctionnement permettant la réalisation des valeurs qu'elle reconnaît. Ainsi, « ces collectifs semblent mimer le geste Constituant, en se dotant d'un espace de délibération sur les règles fondamentales » (Cuillerai 2015). Ce projet éthique s'incarne également dans les processus de définition des critères d'agrément des prestataires et des modalités de sélection de ces derniers (Blanc et Fare 2016). En effet, la réalisation de ce projet passe par l'intégration conditionnelle de prestataires professionnels et de particuliers, tous mis en lien par l'utilisation de la monnaie. Le travail d'élaboration de la charte d'agrément, qui définit ces critères, représente alors un moment essentiel de cette déclinaison concrète de l'orientation politique de la monnaie.

51 Les valeurs définies par la charte se concrétisent ensuite dans les règles de fonctionnement collectives développées par l'organisation: ces règles constituent une deuxième caractéristique d'un commun monétaire. Ces modalités de fonctionnement sont le reflet concret des valeurs fondant l'identité commune, elles doivent en permettre et en favoriser la réalisation, en même temps qu'elles renforcent également la construction de cette identité commune. Le «faire commun » (Bollier 2014) joue ici un rôle fondamental : s'ouvrent à travers les modalités organisationnelles choisies, des espaces politiques de revendication et de défense de ressources jugées comme devant relever des communs, en développant une action collective dans le cadre d'une communauté territoriale autoorganisée et auto-gouvernée régie par des arrangements institutionnels qui lui sont propres (Nyssens et Petrella 2015). Sont ainsi favorisées et valorisées les interdépendances entre les acteurs du territoire dans un cadre démocratique, via leur coopération. Ce qui importe ici, ce sont les pratiques et valeurs sociales développées pour gérer la monnaie de façon partagée afin de favoriser l'émancipation sociale et politique. Cela met en exergue la possibilité de reconnaitre « des modes de régulation démocratique et citoyen des activités économiques" (Fraisse 2003). Ce sont les membres qui vont produire des règles adaptées au contexte local et aux besoins de l'organisation dans une volonté d'agir sur leur territoire autour d'un projet d'utilité sociale. Ces choix, de plus, sont guidés par un projet politique qui débouche sur la volonté d'instaurer un nouveau rapport de souveraineté monétaire par la recherche d'une monnaie répondant, de façon 
plus satisfaisante, aux aspirations et besoins humains, des organisations et/ou des territoires. La création de ces espaces publics, de débats et de co-construction des choix s'inscrit dans le cadre de la "solidarité démocratique» (Laville 2005) et illustre les interdépendances propres à la communauté monétaire ainsi créée. Nous retrouvons ici la notion d'« arènes d'action » d'Ostrom (2011) qui favorisent l'émergence des communs.

Enfin, la mutualisation de la ressource constitue la troisième caractéristique des communs monétaires. En effet, le marché (ou le principe de concurrence si l'on suit l'approche de Servet) est dans ce cadre soumis au principe du partage dans la mesure où il est encastré institutionnellement par les règles de la communauté monétaire reposant sur la mutualisation d'une ressource. De même, le principe réciprocitaire s'incarne dans la volonté des membres de se positionner en situation d'interdépendances volontaires les uns par rapport aux autres où le "souci d'autrui » (Servet 2013) prend toute sa place en soulignant ainsi l'appartenance des membres à la communauté monétaire.

Une identité commune, la construction de règles collectives et démocratiques et la mise en commun de la ressource monétaire constituent donc les trois caractéristiques permettant l'institution du commun monétaire. Ces trois caractéristiques ne sont pas indépendantes l'une de l'autre, mais au contraire, se renforcent mutuellement. La mise en situation d'interdépendances volontaires est favorisée et générée par la volonté d'un " agir démocratique » collectif et par les règles instituées vis-à-vis de la ressource qui permettent la mutualisation de celle-ci. La volonté de s'auto-organiser permet de générer une communauté autour de l'usage de la monnaie et les règles régissant son usage peuvent reposer sur la logique du partage. C'est donc de la volonté de s'associer qu'émergent les règles du partage. Le « faire commun » repose bien sur la construction de la communauté, laquelle peut développer des pratiques de mise en commun. Il n'en demeure pas moins qu'une des conditions d'émergence du commun est la volonté de s'auto-organiser, de coopérer, volonté qui dépend des liens sociaux existants et des règles en vigueur. Ceux-là génèrent la communauté et c'est elle qui permet et qui met en œuvre le partage de la ressource. Par conséquent, c'est l'intrication entre des règles collectives et la construction de l'identité commune qui permet de favoriser la réciprocité entendue comme «une interdépendance pensée comme une complémentarité volontairement instituée d'éléments socialement construits pour être distincts sans s'opposer. Chacun ne pense pas comme un individu, mais agit en tant qu'élément d'un but. Le principe de réciprocité institue donc un passage du collectif à l'individuel et de l'individuel au collectif » (Servet 2013). Du caractère démocratique des règles peut ensuite émerger des logiques de partage pouvant instituer le faire commun.

Nous l'abordons maintenant du point de vue pratique dans ce qui suit.

\subsection{Institution du commun monétaire}

Après avoir précisé notre conceptualisation théorique du commun monétaire, nous pouvons maintenant en envisager les modalités de sa mise en œuvre concrète. Quelques auteurs ont déjà entamé cette réflexion sur ce que seraient des communs - non pas forcément monétaires dans un premier temps - mais des communs financiers, notamment à partir du cas du microcrédit et des modalités de sa gestion. Meyer (2012) a étudié le cas des banques communautaires de développement brésiliennes, et notamment de la Banque Palmas à Fortaleza, à l'aune du cadre d'Ostrom (2010). Cette banque ${ }^{5}$, qui n'en a que le nom (elle n'a pas de licence bancaire), mais qui est le fruit de l'organisation 
en grande partie informelle des habitants de cette zone pauvre, s'inscrit dans une démarche de finance solidaire (Ferraton 2006) et propose une gamme de microcrédits articulés autour d'une monnaie sociale, le Palmas.

L'étude de ce dispositif révèle ainsi un certain nombre de caractéristiques qui suivent les principes partagés des communs tels qu'identifiés par Ostrom (1990). La ressource est limitée au capital financier mobilisé par la banque, et son accès est ouvert à tout habitant du quartier. Les règles d'accès à la ressource sont adaptées aux conditions locales: le microcrédit est rendu accessible aux populations pauvres, sans besoin de contrepartie ou sur la base de leur seul capital social; les agents de crédit employés par la banque exercent une surveillance sur la ressource, et mettent en œuvre des sanctions graduelles lorsqu'une volonté de non-remboursement se manifeste de la part d'un emprunteur. Quand bien même ces mécanismes existent, les conditions de remboursement peuvent être renégociées au cas par cas. Enfin, le «forum économique local», réunissant les citoyens, les représentants des institutions locales et ceux de la banque, réunit l'ensemble des parties prenantes autour des décisions relatives aux orientations de la banque. Ainsi, « le microcrédit productif et la monnaie sociale sont donc administrés selon les principes de ressources communes, c'est-à-dire qu'ils sont considérés comme des biens communs sociaux par la Banque Palmas » (Meyer 2012, p. 93).

C'est bien du renouvellement de la ressource financière dont il est dans ce cas question: elle est « renouvelable uniquement dans la mesure où les prêts accordés sont remboursés, et les coûts de gestion garantis par les taux d'intérêt; autrement dit, la ressource est renouvelée dès lors que le capital retourne dans les caisses de la banque. Quant à la monnaie sociale, son renouvellement est tributaire de son utilisation et de la soutenabilité de son système de gestion » (ibid. p. 15). Les ressources de la banque sont en effet majoritairement constituées de celles qu'elle arrive à mobiliser auprès des banques nationales, et l'émission de la monnaie sociale est tributaire de la constitution et de l'entretien d'un fonds de réserve. Mais c'est bien les pratiques mises en œuvre dans la gestion de ces ressources qui en font un commun : « ce capital dont dispose la BP n'est pas une ressource purement commune dans la mesure où elle découle de contrats avec des entreprises extérieures. C'est donc son utilisation par la BP qui en fait une ressource commune » (ibid. p. 23). Hudon et Meyer (2016) concluent que la monnaie et le crédit, qui peuvent avoir les caractéristiques de biens privés, sont transformés à travers leur mobilisation par les banques communautaires de développement, grâce à l'espace public local de délibération qu'elles instituent, en en faisant des biens hybrides partageant des caractéristiques propres aux communs.

Servet $(2015 a ; 2015 b)$, dans une réflexion plus générale sur le microcrédit comme commun, note également que «la condition première du renouvellement des prêts est leur remboursement ", et que « les charges financières acquittées, à travers les intérêts et commissions, dégagent un accroissement de la ressource». C'est donc d'une intermédiation financière en commun dont traitent ces auteurs. Cette première dimension est néanmoins déjà importante puisqu'elle rejoint les questions de financement et de ses modalités. Paranque (2016), en envisageant une « finance comme commun", relève bien que sur cette question, "deux types de relations sont alors envisageables selon que l'on gère la relation de financement comme un pur risque, c'està-dire comme un calcul de probabilité de défaut et donc fondé sur le prix, ou comme un construit social dans la durée, fondé sur un ensemble d'engagements communs, et donc 
dans une gestion collective de l'information, c'est-à-dire de l'incertitude ». C'est bien la capacité de nouer des relations d'engagement qui est ici en jeu.

Les processus monétaires sont néanmoins plus larges que cette seule intermédiation dès lors qu'on ne s'en tient pas à une logique d'épargne préalable. Il devient alors nécessaire d'inclure dans l'analyse les modalités d'émission de la monnaie. Penser ce que serait un véritable commun monétaire implique de penser une création monétaire en commun. Nous souhaitons ici envisager cette mise en commun de la monnaie à travers les pratiques actuelles des monnaies associatives et de leurs évolutions potentielles à l'aune des réflexions en cours ou des innovations en développement.

Nous avons déjà souligné les deux dimensions de la monnaie qui devraient relever d'une mise en commun pour Giraud (2013) : la liquidité et le financement. La liquidité d'abord, est préservée tant que la monnaie ne fait pas l'objet de l'appropriation privative dont elle peut faire l'objet du fait de son ambivalence. La liquidité est donc garantie tant que la monnaie est utilisée dans le cadre des échanges, autrement dit qu'elle circule. Les monnaies associatives visent d'abord à favoriser cette circulation: en cantonnant une partie de la monnaie nationale au territoire local d'une part, et à une certaine sphère des échanges d'autre part, cette monnaie doit rester liquide. La possibilité d'appropriation privative de cette monnaie est réduite puisqu'elle n'est plus un support de réserve de valeur. En plus de ne pouvoir être reconvertis (autrement que contre des biens et services offerts par le réseau des prestataires membres pour les particuliers) ${ }^{6}$, les billets des monnaies associatives sont en général soumis à une date d'expiration. Puisqu'ils ne peuvent que servir les échanges, ils devraient également rejoindre la circulation plus vite après chaque dépense. Leur vitesse de circulation potentiellement plus élevée amène alors une liquidité supplémentaire au territoire même sans que le volume de monnaie n'ait été augmenté. Dans certains cas, la thésaurisation est combattue de façon active à travers l'instauration d'un principe de fonte: les avoirs monétaires perdent alors périodiquement une fraction de leur valeur, chaque moyen de paiement devant ensuite être réactivé par le paiement de frais proportionnels à la valeur du moyen de paiement. On constate néanmoins qu'il est rarement choisi de mettre en œuvre cette disposition. En complément de ces modalités, il peut aussi être décidé d'imposer des taxes de reconversion pour les professionnels qui souhaiteraient sortir leurs avoirs du réseau de circulation. Cette disposition peut s'avérer inutile si les professionnels s'inscrivent dans une démarche volontariste et trouvent à réemployer la monnaie qu'ils reçoivent (par exemple à travers le versement de salaires ou le paiement de leurs fournisseurs). La fonte et les taxes, si elles sont mises en œuvre, constituent pour l'association émettrice une source de revenus, qui peuvent ensuite servir à la conduite du projet ou être affectés à des objets particuliers en fonction des choix de la structure. Elles peuvent, par exemple, financer une bonification à l'entrée (un bonus est offert à la conversion), activant ainsi le principe de redistribution de Polanyi selon des règles définies collectivement.

61 Si la monnaie peut être un commun, l'une des spécificités principales du commun monétaire est donc bien que, à défaut de restreindre l'accès à la ressource comme dans le cas des communs environnementaux par exemple, «les appropriateurs sont mêmes incités à prélever la ressource commune pour l'utiliser " (Meyer 2012, p. 59). Ainsi, le partage repose sur la circulation de la monnaie: elle est vouée à être dépensée et à circuler au sein de la communauté monétaire, mais cela n'est que le reflet des dettes tissées entre les acteurs de la communauté, et du partage d'une ressource dans une logique réciprocitaire. En suivant le concept de «common pool resources» (Ostrom 2010) 
désignant un ensemble ou un système de ressources qui, d'une part, ne sont pas séparables dans l'usage qui en est fait (idée de « pool » ou de «système ») et, d'autre part, qui sont utilisées par un groupe ou une communauté d'usagers, il est possible que les systèmes de ressource fassent l'objet d'une propriété commune, tandis que les unités de ressource prélevées le soient de façon privative. Mais cet usage privé de la ressource reste subordonné à sa gestion collective.

Concernant le financement, ensuite, les monnaies associatives n'y participent pour l'instant que de façon marginale. En effet, elles sont émises sur la base de la conversion volontaire de la monnaie officielle de la part de leurs utilisateurs. Les euros collectés forment alors un fonds de réserve géré par une banque partenaire. Ces fonds étaient jusque-là principalement gérés par la banque comme tous les autres fonds dont elle dispose, mais sont de plus en plus gérés sur la base d'un partenariat rapproché entre l'association émettrice de la monnaie et la banque. La NEF a par exemple lancé le projet «Prêt de chez moi ", dans le cadre duquel sont financés des projets économiques à plusvalue écologique, sociale et/ou culturelle. L'épargnant ouvre un compte à terme d'une durée de 3 à 7 ans, « rémunéré à $0 \%$ en solidarité avec le projet choisi (qui bénéficiera, lui aussi, d'un prêt à $0 \%$ ) $»^{7}$. C'est l'épargnant qui choisit à quels projets son épargne doit servir. Dans le cas des monnaies associatives, le fonds de réserve peut être mobilisé de la même façon et c'est l'association et ses membres qui choisissent à quels projets, sur le territoire de circulation de la monnaie et en lien avec ses objectifs, seront alloués ses fonds.

D'autres acteurs financiers peuvent également s'inscrire dans une démarche de finance solidaire en partenariat avec les associations émettrices de monnaies. C'est le cas par exemple au pays basque du fonds d'investissement Eusko-Herrikoa. Ici, la société de capital-risque Herrikoa abonde un fonds d'un montant égal au montant de monnaie associative en circulation. Les fonds sont ensuite investis dans des entreprises dont l'activité va dans le sens des objectifs de l'association émettrice de la monnaie. Dans ce cas par exemple, les fonds sont investis dans « des projets d'intérêt collectif ayant pour objectif la relocalisation de l'économie, la promotion de l'euskara, le progrès social et/ou la protection de l'environnement $»^{8}$.

64 À travers ces exemples, on voit la création de circuits financiers courts, à travers lesquels une partie de la ressource financière est mise en commun et gérée en fonction des objectifs du collectif qui est à l'origine de cette ressource.

Si cette mise en commun est aujourd'hui limitée par les contraintes légales actuelles, on peut en envisager l'évolution dans le sens d'un approfondissement de la logique de partage de la ressource monétaire. Au-delà de la gestion par le collectif institué du fonds de réserve, ce fonds pourrait être multiplié de façon à autoriser une émission supérieure à la stricte quantité d'euros collectés, sur le modèle des réserves fractionnaires. Enfin, reconnaître la pertinence de l'action de ces groupes en faveur de l'économie territoriale, ce serait leur permettre une forme d'autonomie monétaire. «Si nous admettons que la monnaie (la liquidité et le crédit) ne devrait être ni un bien privé ni un bien public mais un commun, alors la liberté de création monétaire dont nous disposons devient un atout » (Giraud 2013). La création monétaire est à la base de nos économies monétaires de production. Celle-ci est soutenable dès lors qu'elle répond à un besoin économique, et que ses règles d'émission sont acceptées par tous les membres de la communauté monétaire. Instituer la monnaie en tant que commun permettrait de mobiliser de façon plus large les possibilités de la création monétaire, notamment au profit des enjeux de la transition. Si 
Giraud appelle à la création d'une chambre budgétaire européenne assurant la gestion démocratique d'une BCE mise au service des orientations de long terme de l'économie, nous pensons que les collectifs citoyens réunis autour de modalités de gouvernance adéquats sont tout autant légitimes pour le pilotage d'une émission monétaire territorialisée. Ces collectifs disposent en effet d'une connaissance importante quant au tissu socio-économique local, et sont à même d'identifier les besoins de financements qui participeraient de la création de circuits économiques relocalisés et durables. Cette décentralisation s'insérerait alors dans une forme de subsidiarité monétaire (Fare 2011) à travers laquelle les enjeux locaux pourraient trouver à être réconciliés avec ceux de niveaux supérieurs. La mise en œuvre d'un agencement monétaire subsidiaire approprié aux différents niveaux d'organisation socioéconomique permettrait de coupler une approche en termes de complémentarité monétaire à une approche territoriale délimitant des niveaux d'action pertinents et résultant de la capacité de chaque niveau à inventer des solutions adéquates pour instituer des communs monétaires. Leur développement peut résulter des niveaux territoriaux de décision multiples (des plus territorialisés vers le niveau global), combinant également des organisations diverses et partenariales (privées, publiques, communautaires, etc.) supposant - mais pouvant favoriser - une capacité de participation citoyenne auto-organisée forte.

L'idée n'est donc pas ici de favoriser une « ivresse monétaire » à travers l'émergence de monnaies multiples et indépendantes, mais de tendre vers une forme de fédéralisme monétaire avec « une unité de compte unique qui unifie une pluralité de monnaies de paiement » (Théret 2014). L'unité de compte en constituerait alors le commun des communs . L'unicité de l'unité de compte d'un espace national ou supranational permet «l'expression quantitative des relations sociales » (Aglietta 2016) au sein de cet espace et la monnaie y demeure un « rapport d'appartenance sociale » (ibid.). De la même manière que les communs traditionnels ne visent pas à s'affranchir de l'État (mais plutôt à le compléter), ce n'est pas plus le cas des monnaies associatives qui se définissent d'ailleurs bien comme « complémentaires » à la monnaie nationale. Sans remettre frontalement en cause le pouvoir de l'État vis-à-vis de l'institution monétaire', les monnaies associatives s'en réapproprient une part, sans chercher à s'affranchir de l'espace politique et monétaire dans lequel elles s'inscrivent.

67 Ainsi, les monnaies associatives donnent à voir que la souveraineté monétaire est polysémique, et qu'il faut en distinguer les différentes dimensions. Elle peut effectivement s'affirmer par la définition de l'unité de compte, donc par l'édiction de ce qui acquiert le statut de «monnaie de cours légal». Mais la souveraineté ne se résume pour autant pas à cette seule dimension. La monnaie procède d'une unité de compte mais aussi d'un ensemble de règles, de normes et de conventions liées à son usage. C'est vis-àvis de ces autres dimensions que les monnaies associatives vont réintégrer des formes de souveraineté citoyenne, dans la mesure où elles constituent un saisissement participatif local de certains des enjeux monétaires. Les conditions de l'établissement de l'unité de compte nationale ne sont donc pas modifiées par l'existence ou non des monnaies associatives. Cette souveraineté citoyenne ne vise pas à amener de la fragmentation dans l'espace monétaire national : il s'agit d'une souveraineté dite subsidiaire (Fare 2018). Les différents niveaux de la souveraineté monétaire sont imbriqués, et des acteurs citoyens peuvent y créer des espaces subordonnés, dans lesquels ils établissent des règles propres, sans que cela constitue un acte de sécession. Cette pluralité des formes monétaires, des émetteurs, des souverainetés, caractérise alors une gouvernance polycentrique (Ostrom 
2011). Émergeraient ainsi des centres de décision multiples, indépendants mais partageant des règles communes.

\section{Conclusion}

la norme de la souveraineté monétaire et le statut des monnaies nationales, toujours considérées, dans les textes de loi, comme souveraines et exclusives ${ }^{10}$. Les systèmes monétaires nationaux ou supra-nationaux contemporains sont en effet caractérisés par une conception unitaire de la monnaie et une organisation hiérarchisée autour d'une autorité monétaire centrale chargée de la règle d'émission (convertibilité des monnaies privées en monnaie centrale) et de la stabilité du système. Historiquement, cette conception monétaire homogène et unitaire a fait l'objet de contestations et de réalités contrastées, mais celles-ci sont jusqu'ici largement restées marginales, tant la force de rappel de la souveraineté monétaire centralisée semble puissante. Cependant, à travers les monnaies associatives, émergent des formes de souverainetés non étatiques et reposant sur des groupes institués de la société civile. Ces groupes engendrent alors des souverainetés subsidiaires qui ne visent pas à renverser la souveraineté monétaire nationale, mais plutôt à la compléter à différentes échelles territoriales (des échelles fortement localisées aux moins localisées). Reconnaître ces différents niveaux d'action et la pertinence de différents acteurs à ces différents niveaux mènerait alors à la génération de souverainetés imbriquées, potentiellement capables de répondre de façon plus large aux besoins à différentes échelles, et ainsi de réconcilier ces différentes échelles.

L'agencement des règles d'émission, des instruments de paiement, des modes de gouvernance et de leurs rapports aux pouvoirs publics n'est pas neutre et détermine le sens de l'action et plus globalement l'inscription politique des monnaies. Parce que les caractéristiques organisationnelles résultent d'une «vision politique du rôle de la monnaie » (Blanc 2006b), c'est-à-dire de choix politiques, elles doivent donc être définies collectivement à travers un processus continu de délibération sur les règles et valeurs au fondement du dispositif. La mise en commun de la monnaie que nous avons explorée dans ce texte met en œuvre le principe de partage communautaire (la communauté n'étant ici pas basée sur les appartenances individuelles mais sur la réciprocité et la solidarité entre ses membres) par la création d'une identité commune et le déploiement d'une action collective dans le cadre d'une communauté territoriale auto-organisée et auto-gouvernée, régie par des arrangements institutionnels démocratiques qui lui sont propres, constituant la base de la communauté monétaire. L'organisation sociale repose dans ce cas sur une communauté monétaire développant des logiques marchandes mais subordonnées au partage et s'articulant à la réciprocité. En rejoignant l'analyse de la pluralité institutionnelle, les monnaies associatives, par les règles d'usage et de gouvernance qu'elles développent, peuvent caractériser ce partage communautaire.

70 L'exploration qui a été menée des potentiels approfondissements de la logique de partage au sein des communs monétaires, ici à partir du cas des monnaies associatives, amène à envisager le renforcement des modalités de mise en commun de la liquidité et des possibilités de financement à travers l'emploi du fonds de réserve pour le financement d'activités à plus-value sociale ou environnementale, voire de l'émission monétaire audelà de la stricte quantité d'euros collectés. La mise en pratique de ces pistes pourrait passer par une expérimentation plus grande au sein des communs monétaires, en 
complément des politiques de développement économique, social et environnemental actuellement mises en œuvre.

Dans le contexte actuel de crise systémique et multidimensionnelle, il est plus que temps de repenser l'organisation monétaire en reconnaissant non seulement la permanente pluralité de la monnaie mais également son institutionnalisation souhaitable en tant que commun, si celle-ci doit contribuer à la nécessaire transition de nos économies. Ce contexte de crise est en premier lieu une crise du vivre ensemble : l'oubli de l'inscription de chacun au sein d'une collectivité permettant la persistance des inégalités sociales, des dommages environnementaux ou des atteintes à la démocratie. L'enjeu est alors de réapprendre à vivre ensemble et le commun en est le moyen pertinent. La monnaie devrait être le premier de ces communs qu'il reste à construire.

\section{BIBLIOGRAPHIE}

Aglietta M., P. Ould-Ahmed P. et J.-F. Ponsot, (2016), La Monnaie : Entre dettes et souveraineté, Paris, Odile Jacob.

Aglietta M. et A. Orléan (dir.), (1998), La monnaie souveraine, Paris, Odile Jacob.

Aglietta M., J. Andreau, M. Anspach M., J. Birouste, J. Cartelier, Coppet D. de, C. Malamoud, A. Orléan, J.-M. Servet, B. Théret B. et J.-M. Thiveaud, (1998), « Introduction collective », in M. Aglietta et A. Orléan (dir.), (1998), La monnaie souveraine, Paris, Odile Jacob, p. 9-31.

Amato M., (2016), « La monnaie comme bien commun ? Histoire, théorie, pratiques et enjeux des systèmes de compensation », 12th AFD International Conference on Development : " Commons and Development ", Paris.

Arnsperger C., (2015), « Revenu de base, économie soutenable et alternatives monétaires », L'Économie politique, $\mathrm{n}^{\circ} 67$, p. 34-49.

Baronian L. et C. Vercellone, (2015), « Monnaie du commun et revenu social garanti », Terrains/ Théories, $\mathrm{n}^{\circ} 1$.

Bell S., (2001), " The role of the state and the hierarchy of money ", Cambridge Journal of Economics, 25(2), p. 149-163.

Benes J. et M. Kumhof, (2012), « The Chicago Plan Revisited », IMF Working Paper, WP/12/202.

Black F., (1970), « Banking and the Interest Rates in a World without Money: The Effects of Uncontrolled Banking ", Journal of Bank Research, 1, n³, p. 9-20.

Blanc J., (1994), « Les citoyens face à la complexité monétaire : le cas de la France sous l'Ancien Régime », De Pecunia, VI(3), p. 81-111.

Blanc, J., (2002), « Invariants et variantes de la souveraineté monétaire : réflexions sur un cadre conceptuel compréhensif », Économies et Sociétés - série Monnaie, ME(4), p. 193-213.

Blanc J., (2006), « Les enjeux démocratiques des dispositifs de monnaies sociales », in:

M. Humbert et A. Caillé (dir.), La démocratie au péril de l'économie, Rennes, PUR (Économie et société), p. 263-275. 
Blanc J., (2009), « Usages de l'argent et pratiques monétaires », in P. Steiner et F. Vatin (dir.), Traité de sociologie économique, Paris, Puf, coll. » Quadrige », p. 649-688.

Blanc J., (2013), « Penser la pluralité des monnaies à partir de Polanyi : un essai de typologie », in I. Hillenkamp et J.-L. Laville (dir.), Socioéconomie et démocratie : l'actualité de Karl Polanyi, Toulouse, Erès, p. 241-270.

Blanc J. et M. Fare, (2012), « Les monnaies sociales en tant que dispositifs innovants : une évaluation ", Innovations, Cahiers d'économie de l'innovation, 2012/2, n 38, p. 67-84.

Blanc J. et M. Fare, (2013), « Understanding the role of governments and administrations in the implementation of community and complementary currencies ", Annals of Public and Cooperative Economics, 84, $n^{\circ}$ 1, p. 63-81.

Blanc J. et M. Fare, (2016), « Turning values concrete: the role and ways of business selection in local currency schemes », Review of social economy, vol. 74, n 3, p. 289-319.

Bollier D., (2014), La renaissance des communs. Pour une société de coopération et de partage, Paris, Éditions Charles Léopold Meyer.

Cagan P., (2008), « Monetarism » in S. N. Durlauf et L. E. Blume (eds), The New Palgrave Dictionary of Economics, $2^{\text {nd }}$ ed., Basingstoke, Nature Publishing Group, p. 677-683.

Coriat B., (dir.), (2015), Le retour des communs. La crise de l'idéologie propriétaire, Paris, Les liens qui libèrent.

Courbis B., É. Froment et J.-M. Servet, (1990), « À propos du concept de monnaie », in Cahiers d'économie politique, $\mathrm{n}^{\circ} 18$. Monnaie métallique et monnaie bancaire, sous la direction de MarieThérèse Boyer-Xambeu, Ghislain Deleplace et Lucien Gillard, p. 5-29.

Cuillerai M., (2015), « Monnaie(s) et politique(s) : y a-t-il une perspective sur la démocratie dans "le pluralisme monétaire" ? , Séminaire « La monnaie entre unicité et pluralité », Paris.

Dardot P. et C. Laval, (2014), Commun. Essai sur la révolution au XXI ${ }^{\mathrm{e}}$ siècle, Paris, La Découverte.

Dissaux T. et M. Fare, (2017), « A Collective Redefinition of Money: The Case of the Local Currency "La Gonette" in Lyon, France », communication lors de la 29th annual SASE conference, Lyon.

Dissaux, T. et C. Meyer, (2016), « L'apport des monnaies sociales à la microfinance : le cas des banques communautaires de développement brésiliennes ", Revue d'Économie Financière, $n^{\circ} 124$.

Fama E. F., (1980), « Banking in the Theory of Finance », Journal of Monetary Economics, vol. 6, p. 39-57.

Fare M., (2011), Les conditions monétaires d'un développement local soutenable: des systèmes d'échange complémentaires aux monnaies subsidiaires, Thèse de doctorat en sciences économiques, Université Lumière Lyon 2, Lyon, $490 \mathrm{p}$.

Fare M., (2018). « Sustainable territorial development and monetary subsidiarity » in G. Gómez (dir.), Monetary Plurality Around the World: Theory and Practice, Place, Routledge, à paraître.

Fare M., C. De Freitas et C. Meyer, (2015), « Territorial development and Community currencies: symbolic meanings in Brazilian Community development banks ", International Journal of Community Currency Research, 2015, vol. 19.

Ferraton C., (2006), « Finance solidaire » in J.-L. Laville et A. D. Cattani (eds), Dictionnaire de l'autre économie, Paris, Folio.

Fisher I., (1936), 100\% Money, New York, Adelphi Co. 
Fraisse L., (2003), « Économie solidaire et démocratisation de l'économie », Hermès, nº 36, p. $137-145$.

França Filho G. C. de, A. Scalfoni Rigo, J. J. Torres Silva et C. Meyer, (2012), « L'enjeu de l'usage des monnaies sociales dans les banques communautaires de développement au Brésil : étude du cas de la Banque Palmas ", Revue internationale de l'économie sociale : Recma, 2012, n³24, p. 70-86.

Friedman M. et A. J. Schwartz, (1987), « Has Government Any Role in Money? », in A. J. Schwartz (ed.), Money in historical perspective, Chicago, University of Chicago Press.

Friedman M., (1959), A program for monetary stability, New York, Fordham University Press. Giraud G., (2013), Illusion financière, Édition revue et augmentée, Ivry-sur-Seine, Éditions de l'Atelier.

Hall R. E., (1981), The Government and the Monetary Unit, Working paper \#159, NBER.

Hayek F., (1976), Denationalisation of Money. The Argument Refined. An Analysis of the Theory and Practice of Concurrent Currencies, London, Institute of Economic Affairs.

Hayek F., (1979), « Toward a Free Market Monetary System », Journal of libertarian Studies, 3(1), p. 1-8.

Hardin G., (1968). « The Tragedy of the Commons ». Science, 162(3859), p. 1243-1248.

Harribey J.-M., E. Jeffers, Marie J., D. Plihon et J.-F. Ponsot, (2018), La Monnaie - Un enjeu politique, Paris, Éditions du Seuil, coll. « Points », 240 p.

Hillenkamp I. (2013), « Le principe de householding aujourd'hui. Discussion théorique et approche empirique par l'économie populaire », in I. Hillenkamp et J.-L. Laville (dir.), $S$ ocioéconomie et démocratie. L'actualité de Karl Polanyi, Toulouse, Érès, p. 215-239.

Hudon M. et C. Meyer, (2016), « A Case Study of Microfinance and Community Development Banks in Brazil Private or Common Goods? », Nonprofit and Voluntary Sector Quarterly, vol. 45, n 4. Keynes J. M., (1930), A treatise on money, New York, Harcourt, Brace and Company.

Knapp G. F., (1924), The State Theory of Money, London, Macmillan \& Company Limited.

Laval C., (2016), " “Commun” et “communauté” : un essai de clarification sociologique », SociologieS, Dossier "Des communs au commun : un nouvel horizon sociologique ?", http:// journals.openedition.org/sociologies/5677

Lerner A. P., (1947), « Money as a Creature of the State », The American Economic Review, 1947, vol. 37, n², p. 312-317.

Mauss M., (1993) [1950], Sociologie et anthropologie, Paris, PUF.

Melo J., E. Becu et C. Freitas de, (2009), Viva Favela ! Quand les démunis prennent leur destin en main, Neuilly-sur-Seine, Michel Lafon, $281 \mathrm{p}$.

Meyer C., (2012), Les finances solidaires comme biens communs durables : étude de cas de la Banque communautaire de développement Palmas (Brésil), Master en sciences de la population et $\mathrm{du}$ développement, Université libre de Bruxelles, Faculté des sciences sociales et politiques.

Nyssens M. et F. Petrella, (2015), « ESS et ressources communes : Vers la reconnaissance d'une diversité institutionnelle. Interpellations croisées ", Revue française de socio-économie, 15(1), p. 117-134.

Orléan A., (1998), « La monnaie autoréférentielle : réflexions sur les évolutions monétaires contemporaines », in M. Aglietta et A. Orléan (dir.), La Monnaie Souveraine. Paris, Odile Jacob. 
Ostrom E., (1990), Governing the commons: the evolution of institutions for collective action, Cambridge, New York, Cambridge University Press, coll. » The Political economy of institutions and decisions ", 280 p.

Ostrom E., (2010), Gouvernance des biens communs : pour une nouvelle approche des ressources naturelles, Bruxelles, De Boeck, coll. » Planète en jeu ».

Ostrom E., (2011), « Par-delà les marchés et les états. La gouvernance polycentrique des systèmes économiques complexes ", Revue de l'OFCE, Débats et politiques, n 120, p. 15-72.

Ould-Ahmed P. et J.-F. Ponsot (dir.), (2015), Dossier « Contestations monétaires : Une économie politique de la monnaie », Revue de la régulation. Capitalisme, institutions, pouvoirs, $\mathrm{n}^{\circ} 18 \mid 2 \mathrm{e}$ semestre / Autumn 2015.

Paranque B., (2016), « Conclusion provisoire. Vers une finance comme "commun” ? » in R. Pérez (dir.), La finance autrement? Réflexions critiques sur la finance moderne. Capitalismes - éthique institutions, Villeneuve d'Ascq, Presses universitaires du Septentrion, p. 293-300.

Polanyi K., (1983) [1944], La grande transformation. Aux origines politiques et économiques de notre temps, Paris, Gallimard.

Polanyi K., (2008a) [1957], « L'économie en tant que procès institutionnalisé », in K. Polanyi, Essais de Karl Polanyi, Paris, Le Seuil, p. 53-77.

Polanyi K., (2008b) [1957], « La sémantique des usages de la monnaie », in K. Polanyi, Essais de Karl Polanyi, Paris, Le Seuil, p. 221-243.

Samuelson P.A., (1954), « The Pure Theory of Public Expenditure », The Review of Economics and Statistics, 36(4).

Selgin G. A. et L. H. White, (1994), « How Would the Invisible Hand Handle Money? », Journal of Economic Literature, American Economic Association, vol. XXXII, Nº 4, (December, 1994),

p. 1718-1749.

Servet J.-M., (2012), Les monnaies du lien, Lyon, PUL.

Servet J.-M., (2013), « Le principe de réciprocité aujourd'hui. Un concept pour comprendre et construire l'économie solidaire », in I. Hillenkamp et J.-L. Laville (dir.) Socioéconomie et démocratie - l'actualité de Karl Polanyi, Érès, Toulouse, p. 187-213.

Servet J.-M., (2015a), La vraie révolution du microcrédit, Paris, Odile Jacob, 256 p.

Servet J.-M., (2015b), « La finance et la monnaie comme un "commun" », Notes de l'Institut Veblen pour les réformes économiques, 2015.

Servet J.-M., (2015c), « Corporations dans l'Europe d'Ancien Régime et principe d'autosuffisance. Comprendre le caractère moral des corporations d'Ancien Régime ", in Economie morale, Morale de l'économie, Paris, L'Harmattan, p. 55-86.

Servet J.-M., (2016), «Institution monétaire et commun(s) », Colloque «Institutionnalismes Monétaires Francophones : Bilan, Perspectives Et Regards Internationaux », Lyon.

Servet J.-M. et S. Swaton, (2017), « Penser la dimension de commun de la monnaie à partir de l'exemple des monnaies complémentaires locales », Revue Interventions économiques, $\mathrm{n}^{\circ} 59$.

Théret, B., (2014), « Sortir d'en bas par le haut de la crise de l'Euro-zone : Une réponse en termes de fédéralisme monétaire ", Revue du MAUSS permanente.

Théret B., (2008), « Les trois états de la monnaie. Approche interdisciplinaire du fait monétaire », Revue Économique, vol. 59, $\mathrm{n}^{\circ}$ 4, p. 813-841. 
Théret B., (dir.), (2007), La monnaie dévoilée par ses crises. Crises monétaires d'hier et d'aujourd'hui, Paris, Éditions de l'EHESS.

Théret B. et M. Zanabria, (2007), « Sur la pluralité des monnaies publiques dans les fédérations. Une approche de ses conditions de viabilité à partir de l'expérience argentine récente », Économie et Institutions, $\mathrm{n}^{\circ}$ 10-11, http://journals.openedition.org/ei/295

Wray L.R., (1990), Money and Credit in Capitalist Economies: The Endogenous Money Approach, Edward Elgar Publishing.

Wray L.R., (2015), « Modern money theory » in Steven N. Durlauf et Lawrence E. Blume (eds.), The New Palgrave Dictionary of Economics, Basingstoke, Palgrave Macmillan.

Zelizer V., (2005) [1994], La signification sociale de l'argent, Paris, Le Seuil.

\section{NOTES}

1. Zelizer (2005 [1994]) apporte une contribution majeure à l'analyse de la pluralité en termes d'usages monétaires différenciés. En étudiant les pratiques monétaires contemporaines, elle illustre l'existence de marquages sociaux, culturels et moraux de l'argent visant à restreindre les usages ou les usagers.

2. Polanyi semble hésiter sur le principe de householding dans la mesure où contrairement aux autres principes d'intégration, ce dernier est évoqué dans La Grande Transformation (1944), puis disparaît dans Les systèmes économiques dans l'histoire et dans la théorie (1957). Ainsi la plupart des auteurs contemporains ne retiennent que trois principes d'intégration.

3. Pour une application empirique au cas de la monnaie associative lyonnaise, la Gonette, voir Dissaux et Fare (2017).

4. Si nous observons cette identité commune dans le cadre des monnaies associatives, cette caractéristique n'est pas propre à ces monnaies. Comme nous l'avons explicité auparavant, toute monnaie renvoie à un collectif.

5. Sur la Banque Palmas, voir par exemple Melo, Becu et de Freitas 2009; de França Filho et al. 2012 ; Fare, De Freitas et Meyer 2015 ; Dissaux et Meyer 2016.

6. Les professionnels membres du réseau ont généralement la possibilité de reconvertir leurs billets de monnaie associative en monnaie officielle moyennant le paiement d'une taxe (cf. infra).

7. https://www.pretdechezmoi.coop/fr/comment-ca-marche/soutenir-un-projet/

8. http://www.euskalmoneta.org/fr/fonds-dinvestissement-eusko-herrikoa/

9. Elles remettent bien davantage en cause le pouvoir acquis par les entités privées auxquelles la gestion de la monnaie a été déléguée, dont notamment les banques commerciales.

10. En France, la loi $n^{\circ} 2014-856$ du 31 juillet 2014 relative à l'économie sociale et solidaire reconnait les monnaies associatives comme «titres de paiement». Leur pleine acceptation, notamment par les finances publiques, reste cependant encore à réaliser. 


\section{RÉSUMÉS}

Si la monnaie est perçue par beaucoup comme devant naturellement constituer un bien public, dans le contexte actuel elle est de moins en moins vue comme permettant le bien commun. Elle fait l'objet de remises en cause et de contestations qui posent - avec une variété de projets pouvant être radicalement différents - la question du mode de gestion et des modalités de gouvernance de cette ressource particulière. Nous verrons d'abord que si la théorie économique aborde quasi exclusivement la monnaie du point de vue du marché ou bien de celui de l'État, la monnaie est avant tout un fait social total. Dépasser l'opposition marché/État relativement à la monnaie permet d'en envisager le caractère commun. La monnaie peut-elle alors être instituée en tant que commun? et que serait un commun monétaire? En prolongeant le principe d'intégration polanyien de householding, nous définissons le principe de partage communautaire, qui permet de conceptualiser le partage d'une ressource monétaire. Nous verrons que les règles établies au sein d'une communauté de paiement permettent d'instaurer la monnaie comme commun.

If money is perceived by many as naturally being a public good, nowadays it is less and less seen as allowing the common good. Money is being questioned and challenged, raising - with a wide variety of projects which can be radically different - the question of how to manage and how to govern this particular resource. First, we shall see that economic theory deals with money almost exclusively from the point of view of the market or of the state. But money is above all a total social fact. Overcoming the market/state opposition with regard to money makes it possible to consider money as a common. Then, can money be instituted as a common and what would be a monetary common? Building on the polanyian integration principle of "householding", we define the principle of community sharing, which makes it possible to conceptualize the sharing of a monetary resource. We will see that the rules established within a payment community make it possible to establish money as a common.

\section{INDEX}

Keywords : money, commons, local currencies, sharing, Ostrom, Polanyi

Mots-clés : monnaie, commun, monnaies locales associatives, partage, Ostrom, Polanyi

Code JEL A13 - Relation of Economics to Social Values, E42 - Monetary Systems • Standards • Regimes • Government and the Monetary System • Payment Systems, H41 - Public Goods, 035 Social Innovation, Z13 - Economic Sociology $\bullet$ Economic Anthropology $\bullet$ Social and Economic Stratification

\section{AUTEURS}

\section{TRISTAN DISSAUX}

Doctorant en sciences économiques, Triangle (UMR 5206), Université Lumière Lyon 2 ;

tristan.dissaux@univ-lyon2.fr 


\section{MARIE FARE}

Maître de conférences en sciences économiques, Triangle (UMR 5206), Université Lumière Lyon 2. m.fare@univ-lyon2.fr 